\title{
A Possible Detection of CO $(J=3-2)$ Emission From the Host Galaxy of GRB 980425 with Atacama Submillimeter Telescope Experiment
}

Bunyo Hatsukade ${ }^{1}$, Kotaro Kohno ${ }^{1}$, Akira Endo ${ }^{1,2}$, Tomoka Tosaki ${ }^{3}$, Kouji Ohta ${ }^{4}$, Seiichi Sakamoto ${ }^{2}$, Nobuyuki Kawai ${ }^{5}$, Juan R. Cortés ${ }^{6}$, Kouichiro Nakanishi ${ }^{3}$, Takeshi Okuda ${ }^{1,3}$, Kazuyuki Muraoka ${ }^{1}$, Takeshi Sakai $^{2}$, Hajime Ezawa ${ }^{2}$, Nobuyuki Yamaguchi ${ }^{2}$, Kazuhisa Kamegai ${ }^{1}$, and Ryohei Kawabe ${ }^{2}$

${ }^{1}$ Institute of Astronomy, the University of Tokyo email: hatsukade@ioa.s.u-tokyo.ac.jp

${ }^{2}$ National Astronomical Observatory of Japan

${ }^{3}$ Nobeyama Radio Observatory

${ }^{4}$ Department of Astronomy, Kyoto University

${ }^{5}$ Department of Physics, Tokyo Institute of Technology

${ }^{6}$ Departamento de Astronomia, Universidad de Chile

\begin{abstract}
Long-duration gamma-ray bursts (GRBs) are considered to be due to the death of massive stars. Therefore, GRBs are closely associated with the star formation of host galaxies. Since GRBs can be detected at cosmological distances, they are expected to be probes of the star formation history of the Universe. In order to determine the use of GRBs, it is essential to understand the star formation of their hosts. Multi-wavelength observations have shown that the star formation rates (SFRs) of GRB hosts derived from submillimeter/radio observations are generally higher than those from optical/UV observations (Berger et al. 2003). This implies that GRB hosts have a large amount of molecular gas and massive star formation obscured by dust. In order to solve this problem, it is necessary to derive the SFRs in a method which is independent of existing methods and not affected by dust extinction.

We observed ${ }^{12} \mathrm{CO}(J=3-2)$ line emission from the host galaxy of GRB 980425 using the Atacama Submillimeter Telescope Experiment (ASTE). Five points were observed covering the entire region of the galaxy, and we find possible emission features $(\mathrm{S} / \mathrm{N} \sim 3 \sigma)$ at the velocity range corresponding to the redshift of the galaxy. By combining all spectra of five points, we obtain a global spectrum with a $\sim 4 \sigma$ emission feature. If the features are real, this is the first detection of $\mathrm{CO}$ among GRB hosts. We derive the total gas mass of $M\left(\mathrm{H}_{2}\right)=7 \pm 2 \times 10^{8} M_{\odot}$ assuming a CO-to- $\mathrm{H}_{2}$ conversion factor of $\alpha_{\mathrm{CO}}=8.0 M_{\odot}\left(\mathrm{K} \mathrm{km} \mathrm{s}^{-1} \mathrm{pc}^{2}\right)^{-1}$, which is deduced using the correlation between the $\alpha_{\mathrm{CO}}$ and the metallicity. The dynamical mass is calculated to be $M_{\mathrm{dyn}}=2 \times 10^{10} M_{\odot}$, and $M\left(\mathrm{H}_{2}\right) / M_{\mathrm{dyn}} \sim 3 \%$ is consistent with those of nearby dwarfs and normal spirals. The derived SFR is $0.5 \pm 0.1 M_{\odot} \mathrm{yr}^{-1}$ based on the Schmidt law. This SFR agrees with the results of previous $\mathrm{H} \alpha$ observations, suggesting that there is no significant obscured star formation in this host galaxy. This result implies that there is a variety of GRB hosts in terms of the presence of obscured star formation.
\end{abstract}

\section{References}

Berger, E., et al. 2003, ApJ, 588, 99.

Hatsukade, B., et al. 2006, submitted to PASJ. 